\title{
Avaliação comparativa da neoformação óssea em implantes imediatos associados ao \\ levantamento de seio maxilar bilateral preenchido com coágulo sanguíneo com ou sem osso autógeno
}

Comparative evaluation of boné neoformation in immediate implants associated with the lifting of a bilateral maxillary sinus filled with a blood clot with or without autogenous boné

Evaluación comparativa de la neoformación ósea en implantes inmediatos asociados al levantamiento de un seno maxilar bilateral relleno de un coágulo de sangre con o sin hueso autógeno

Recebido: 08/04/2021 | Revisado: 17/04/2021 | Aceito: 23/04/2021 | Publicado: 09/05/2021

Ana Maira Pereira Baggio
ORCID: https://orcid.org/0000-0002-9474-5091
Universidade Estadual Paulista, Brasil
E-mail: anamairabaggio@ gmail.com
Juan Marcelo Lassalle Olivera
ORCID: https://orcid.org/0000-0003-3399-4980
E-mail: juanmarcelolassalle@ @mail.com
Stéfani Caroline Ferriolli
Instituto Latino Americano de Pesquisa e Esino Odontologic, Brasi
ORCID: https://orcid.org/0000-0003-0019-6026
Universidade Estadual Paulista, Brasil
E-mail: stefani.ferriolli @outlook.com
Vinícius Ferreira Bizelli
ORCID: https://orcid.org/0000-0003-1813-3509
Universidade Estadual Paulista, Brasil
E-mail:viniciusbizelli@gmail.com
Flávia Gasparini Kiatake Fontão
ORCID: https://orcid.org/0000-0002-3924-7743
E-mail: fgaspar@ilapeo.com.br
Ana Paula Farnezi Bassi
Instituto Latino Americano de Pesquisa e Ensino Odontológico, Brasil
ORCID: https://orcid.org/0000-0002-0031-4953
Universidade Estadual Paulista, Brasil
E-mail: ana.bassi @unesp.br

\begin{abstract}
Resumo
A redução da altura óssea em regiões posteriores de maxila é comum em maxilas edêntulas. Pacientes que desejam reabilitação com implantes necessitam realizar tratamentos adicionais para obter ganho ósseo em altura. Entre os tratamentos mais comuns para ganho vertical, o levantamento do seio maxilar com preenchimento da cavidade com biomateriais e/ou enxerto autógeno é a forma mais relatada na literatura. Trabalhos vem demonstrando que o uso de coágulo sanguíneo como material de preenchimento e instalação imediata de implantes possibilita a neoformação óssea e osseointegração. Este trabalho tem por objetivo relatar um caso clínico de levantamento de seio bilateral comparando a forma de preenchimento (osso autógeno vs coágulo) e avaliar aspectos diferenciais na neoformação óssea com implantes imediatos. Realizou-se o procedimento num paciente masculino de 37 anos com ausência dos elementos 16 e 26. Para preenchimento do seio esquerdo utilizou-se osso autógeno particulado e no seio direito o coágulo sanguíneo. Durante 1 ano de acompanhamento realizou-se 5 tomografias computadorizadas que revelaram neoformação óssea nos dois seios maxilares e ausência de colabamento da membrana. Na análise de variação da densidade em função do tempo e tipo de tratamento observou-se uma queda na densidade óssea após o período de um ano em ambos os grupos. Nas análises transversais, observou-se que os dois tratamentos apresentam medidas semelhantes de densidade, indicando que o tratamento experimental pode ser tão efetivo quanto o tratamento controle. Os resultados são considerados positivos levando-se em consideração a simplificação da técnica empregada em comparação com aquela que requer uma área doadora.
\end{abstract}

Palavras-chave: Neoformação óssea; Coágulo sanguíneo; Implante imediato. 


\begin{abstract}
The reduction in bone height in posterior regions of the maxilla is common in edentulous jaws. Patients who wish to undergo rehabilitation with implants need to undergo additional treatments to obtain bone gain in height. Among the most common treatments for vertical gain, lifting the maxillary sinus with filling the cavity with biomaterials and / or autogenous graft is the most reported form in the literature. Studies have shown that the use of a blood clot as a filling material and immediate implant installation enables bone neoformation and osseointegration. This paper aims to report a clinical case of bilateral sinus lift comparing the filling form (autogenous bone vs clot) and to evaluate differential aspects in bone neoformation with immediate implants. The procedure was performed on a 37-year-old male patient with the absence of elements 16 and 26. To fill the left sinus, particulate autogenous bone was used and the blood clot in the right sinus. During 1 year of follow-up, 5 computed tomography scans were performed, which revealed new bone formation in the two maxillary sinuses and the absence of membrane collapse. In the analysis of the variation of density as a function of time and type of treatment, a decrease in bone density was observed after a period of one year in both groups. In cross-sectional analyzes, it was observed that the two treatments have similar measures of density, indicating that the experimental treatment can be as effective as the control treatment. The results are considered positive considering the simplification of the technique used in comparison with that which requires a donor area.
\end{abstract}

Keywords: New bone formation; Blood clot; Implant immediately.

\title{
Resumen
}

La reducción de la altura del hueso en las regiones posteriores del maxilar es común en los maxilares edéntulos. Los pacientes que deseen someterse a rehabilitación con implantes deben someterse a tratamientos adicionales para obtener ganancia ósea en altura. Entre los tratamientos más comunes para la ganancia vertical, la elevación del seno maxilar con relleno de la cavidad con biomateriales y / o injerto autógeno es la forma más reportada en la literatura. Los estudios han demostrado que el uso de un coágulo de sangre como material de relleno y la instalación inmediata del implante permite la neoformación y osteointegración ósea. Este artículo tiene como objetivo reportar un caso clínico de elevación de seno bilateral comparando la forma de obturación (hueso autógeno vs coágulo) y evaluar aspectos diferenciales en la neoformación ósea con implantes inmediatos. El procedimiento se realizó a un paciente masculino de 37 años con ausencia de los elementos 16 y 26. Para el llenado del seno izquierdo se utilizó hueso autógeno particulado y el coágulo sanguíneo en el seno derecho. Durante 1 año de seguimiento se realizaron 5 tomografías computarizadas, que revelaron nueva formación de hueso en los dos senos maxilares y ausencia de colapso de la membrana. En el análisis de la variación de densidad en función del tiempo y tipo de tratamiento, se observó una disminución de la densidad ósea al cabo de un año en ambos grupos. En análisis transversales, se observó que los dos tratamientos tienen medidas de densidad similares, lo que indica que el tratamiento experimental puede ser tan efectivo como el tratamiento control. Los resultados se consideran positivos considerando la simplificación de la técnica utilizada en comparación con la que requiere un área donante.

Palabras clave: Formación de hueso nuevo; Coágulo de sangre; Implante inmediatamente.

\section{Introdução}

A implantodontia consiste em uma especialidade que visa a reabilitação de pacientes que tiveram seus elementos dentários perdidos, sejam eles edêntulos totais ou parciais, buscando devolver função, estética e fonética ao paciente e consequentemente melhor qualidade de vida. Sabe-se que a perda do elemento dentário leva à um processo de reparação e remodelação na região levando à chamada reabsorção óssea (David et al., 2018; Guilherme et al., 2009; Patel et al., 2015; Albuquerque et al., 2014). A maxila apresenta-se como a região mais complexa para a Implantodontia em decorrência dos aspectos estético e funcional, tendo como fatores agravantes a qualidade óssea (baixa densidade), o grau de exposição gengival e a intensa reabsorção notadamente na região anterior. Quando a reabsorção óssea ocorre na região posterior da maxila, é comum que esta seja acompanhada da pneumatização do seio maxilar, o que ocasiona uma redução do assoalho do seio e, consequentemente, a diminuição vertical do remanescente ósseo (David et al., 2018; Patel et al., 2015; Terra et al., 2011; Albuquerque et al., 2014).

Com o intuito de permitir a instalação de implantes osseointegrados nessas regiões, onde o remanescente ósseo encontra-se insuficiente, pode-se lançar mão do levantamento do seio maxilar, que atualmente, pode ser realizado de diferentes maneiras a fim de facilitar a fixação do parafuso de implante e consequentemente, permitir a reabilitação protética (David et al., 2018; Ferreira et al., 2021; Bassi et al., 2015; Guilherme et al., 2009). 
A técnica de levantamento de seio maxilar é o procedimento de aumento ósseo mais realizado e mais previsível para resolução de maxilas atrésicas (David et al., 2018; Albuquerque et al., 2014). A escolha da técnica baseia-se no quanto de levantamento do assoalho será necessário e na estrutura óssea residual remanescente. Pode ser realizada uma abordagem através da confecção de uma janela óssea na parede lateral ou uma abordagem via alveolar (David et al., 2018; Albuquerque et al., 2014).

O uso de coágulo sanguíneo como material de preenchimento na loja criada após a elevação da membrana sinusal e instalação imediata de implantes dentários, possibilita a neoformação óssea e osseointegração simultaneamente (Ferreira et al., 2021; Bassi et al., 2015; Terra et al., 2011; Thor et al., 2007; Kim et al., 2010).

Quanto aos materiais que podem ser empregados para o preenchimento em levantamento de seio maxilar, podem ser citados os enxertos autógenos (aqueles em que o doador e o receptor são o mesmo indivíduo), homôgenos (indivíduos da mesma espécie), heterógenos (indivíduos de espécies diferentes), alógenos (quando o material de enxerto é de origem mineral ou sintética), bem como combinações desses materiais (Guilherme et al., 2009; Kürrkçü et al., 2005; Albuquerque et al., 2014; Fouad et al., 2018).

Apesar do osso autógeno ser considerado a melhor opção em comparação aos substitutos ósseos devido à sua alta biocompatibilidade e potencial de osteoindução e osteocondução, a morbidade da área doadora, o aumento do tempo cirúrgico e custos adicionais devem ser levados em consideração. Ademais, deve-se lembrar que os enxertos autógenos são limitados em quantidade, ocasionam certa morbidade na área doadora e necessitam de um tempo cirúrgico maior para sua remoção (Guilherme et al., 2009; Kürrkçü et al., 2005; Burchardt et al., 1983).

A literatura (Thor et al., 2007; Chen et al., 2007; Kim et al., 2020; Betoni Junior et al., 2012) têm confirmado uma técnica para elevação do seio maxilar com instalação imediata de implantes e o uso de coágulo sanguíneo como material de preenchimento entre a membrana sinusal, o implante dentário e as paredes do seio maxilar. A confirmação experimental do potencial osteogênico da membrana sinusal, os fatores de crescimento e diferenciação de células ósseas presentes no coágulo sanguíneo e os princípios estabelecidos de regeneração tecidual guiada tornam a técnica do coágulo vantajosa e previsível quando respeitadas as suas limitações (Ferreira et al., 2021; Bassi et al., 2015; Thor et al., 2007; Chen et al., 2007; Betoni Junior et al., 2012; Srouji et al., 2009).

\section{Metodologia}

Este estudo trata-se de um relato de caso de forma qualitativa e descritiva (Pereira et al., 2018), trazendo dados relevantes da literatura a respeito da neoformação óssea correlacionada ao caso clínico apresentado de um paciente do sexo masculino, de 37 anos de idade, submetido ao levantamento de seio maxilar bilateral com implantes imediatos, utilizando o coágulo como material de preenchimento com e sem osso autógeno particulado.

\section{Caso Clínico}

Paciente do sexo masculino, 37 anos de idade, leucoderma, compareceu ao Instituto Latino Americano de Ensino e Pesquisa Odontológica - ILAPEO, queixando-se de função mastigatória comprometida devido à ausência dos elementos $16 \mathrm{e}$ 26. Foi realizada a anamnese juntamente com os exames laboratoriais, radiografia panorâmica e tomografia computadorizada, os quais não constataram nenhuma contraindicação para a realização do tratamento odontológico. Apesar do tempo da perda dos elementos ser superior a 5 anos, ao exame clínico constatou-se manutenção do espaço protético (Figura 1A). Visto que o espaço mesio-distal de ambas as áreas era próximo de $9 \mathrm{~mm}$, foi proposta a reabilitação dos elementos unitários através de implantes e coroas metalo-cerâmicas. Entretanto, após avaliação dos exames de imagem (Figuras 1B, C e D), ficou evidente a necessidade de enxerto ósseo de levantamento do seio para ganho de altura óssea e instalação de implantes com comprimentos 
compatíveis com o tipo de osso e sua densidade. Como o remanescente de rebordo ósseo era próximo de $6 \mathrm{~mm}$ de ambos os lados, optou-se por se realizar o procedimento de enxertia e concomitante instalação imediata dos implantes.

Figura 1. A. Aspecto clínico oclusal intraoral. B. Imagem da radiografia panorâmica. C. Aspecto tomográfico do seio da região do 16. D. Aspecto Tomográfico do seio da região do 26.
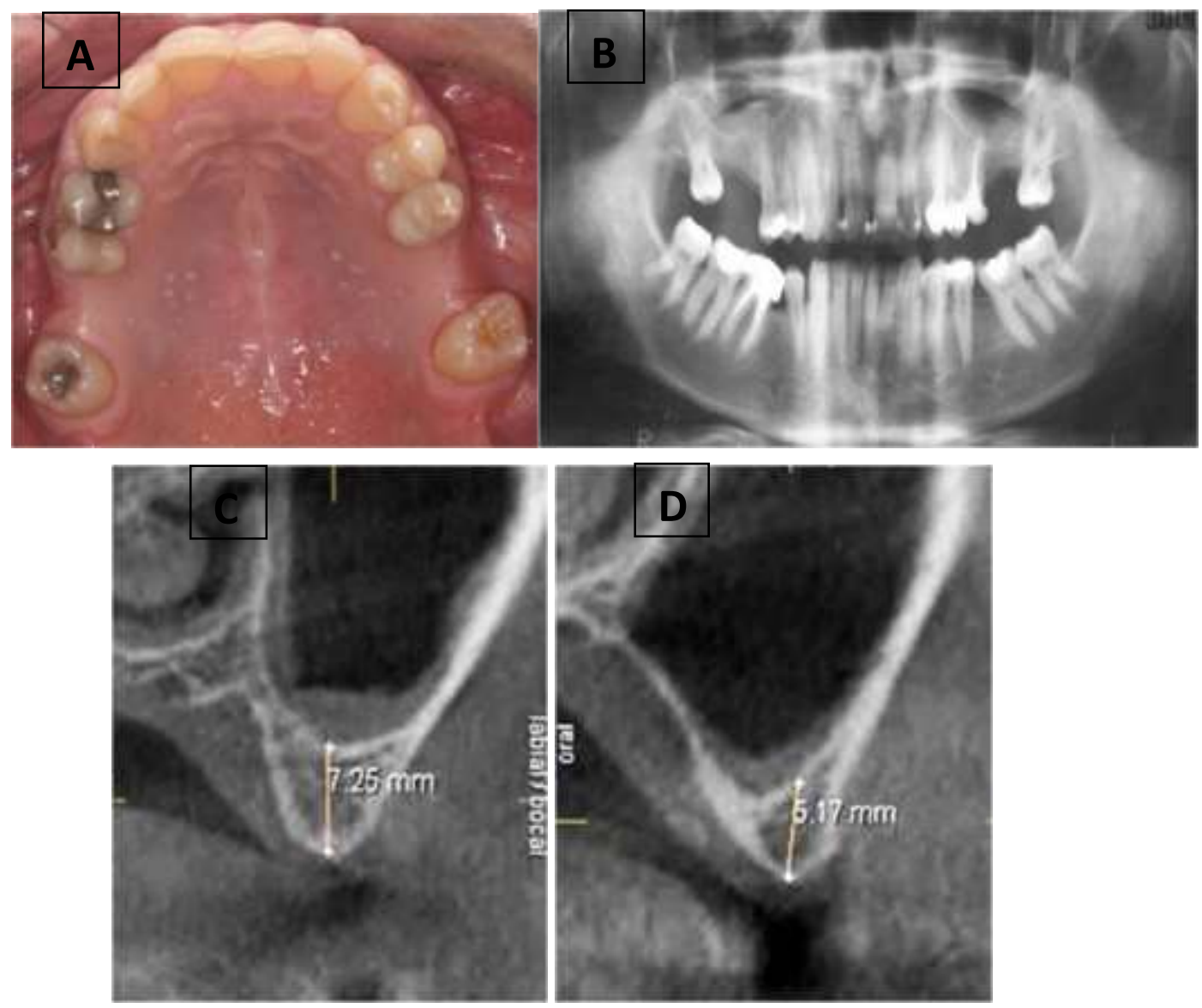

Fonte: Autores.

A região do elemento 26 (Figura 2A) apresentava um remanescente residual próximo de $5 \mathrm{~mm}$ e foi proposta a técnica de levantamento da membrana sinusal com preenchimento da cavidade com osso autógeno particulado. A região do elemento 16 (Figura 3A) possuía aproximadamente $7 \mathrm{~mm}$ de remanescente ósseo, sendo a mais indica técnica da elevação da membrana sinusal com preenchimento por coágulo sanguíneo e instalação imediata do implante. Após diagnóstico, plano de tratamento e consentimento do paciente a cirurgia foi realizada. O paciente recebeu orientações impressas sobre cuidados pré-operatórios e pós-operatórios e uma hora antes da cirurgia foi medicado com $10 \mathrm{mg}$ de Diazepam e $4 \mathrm{mg}$ de Dexametasona.

Iniciou-se a cirurgia abordando o seio maxilar esquerdo realizando a anestesia local, seguida de uma incisão de Novak da região do elemento 25 até o elemento 27. Foi efetuado o descolamento mucoperiostal total do retalho, seguido da confecção de uma janela na parede lateral do seio maxilar (Figura 2A), realizada com brocas diamantadas esféricas montadas e irrigação abundante. Constatado o desprendimento da janela óssea da parede lateral do seio, foram utilizadas curetas sinusais para a liberação e a elevação da membrana sinusal. Foi efetuada uma elevação aproximada de $8 \mathrm{~mm}$ da membrana, para que se pudesse instalar um implante de $13 \mathrm{~mm}$ de comprimento (Figura 2B). Após elevação da membrana sinusal da região do elemento 26, foi abordada a área doadora, sendo esta a linha oblíqua externa esquerda da mandíbula. Após anestesia por bloqueio regional, uma incisão foi realizada sobre a linha oblíqua. O descolamento do periósteo da região foi realizado 
seguindo-se as osteotomias horizontal e vertical. Após a remoção do bloco, foi colocado o enxerto em água estéril. A ferida foi suturada com fio de nylon 5.0. O bloco removido foi fracionado e particulado com particulador ósseo da marca Neodent@.

Como a proposta era enxertia com instalação imediata de implante, foi selecionado um implante cônico "Alvim Cone Morse" da marca Neodent ${ }^{\circledR}$, com dimensões 4,3 x $13 \mathrm{~mm}$ (Figura 2C). Antes da instalação do implante foi inserido osso particulado próximo à parede medial do seio maxilar. Após a instalação do implante, o qual ficou com sua porção cervical 1 mm infra ósseo, completou-se o preenchimento da cavidade com osso particulado (Figuras 7). O retalho foi reposicionado e suturado com fio mononylon 5.0.

Figura 2. A. Levantamento do seio lado esquerdo. B. Instalação do implante. C. Preenchimento do seio com osso autógeno particulado.

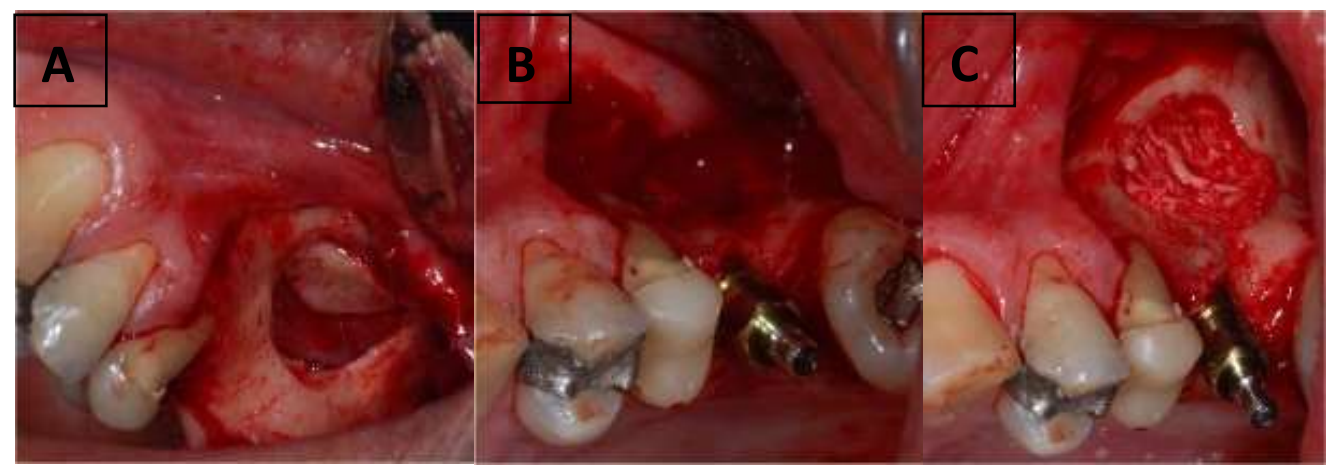

Fonte: Autores.

A abordagem da região do elemento 16 teve as mesmas características adotadas na região do 26. Após anestesia local infiltrativa foi realizada uma incisão biangular baixa (Novak) entre os dentes 15 a 17. Descolamento mucoperiostal total foi efetuado, seguindo a sequência de realização da osteotomia da parede lateral do seio maxilar e descolamento da membrana sinusal acima descrita (Figura 3A). Foi elevado aproximadamente $8 \mathrm{~mm}$ da membrana, para que se pudesse instalar um implante de $13 \mathrm{~mm}$ de comprimento (Figura 3B).

Nessa região do elemento 16 optou-se pelo preenchimento da cavidade no seio apenas com coágulo sanguíneo. Já que a região não recebeu nenhum acréscimo ósseo ou de biomaterial, seguiu-se diretamente à fresagem óssea e à instalação do implante. Assim como na região do elemento 26, foi selecionado um implante cônico "Alvim Cone Morse" da marca Neodent ${ }^{\circledR}$, com dimensões 4,3 x 13 mm. Após a instalação do implante foi observado o completo preenchimento de sangue da cavidade criada. A janela óssea que foi rotacionada, estabeleceu o novo limite para o assoalho do seio maxilar, e foi mantida de maneira horizontal pelo ápice do implante. A entrada da loja foi protegida com uma membrana biológica do tipo cortical bovina de nome Genderm ${ }^{\circledR}$ e fabricada pela Genius (Figura 3C). O retalho foi reposicionado e suturado com fio mononylon 5.0 . 
Figura 3. A. Levantamento do seio lado direito. B. Instalação do implante. C. Preenchimento do seio com coágulo e membrana de cortical bovina na parede anterior.

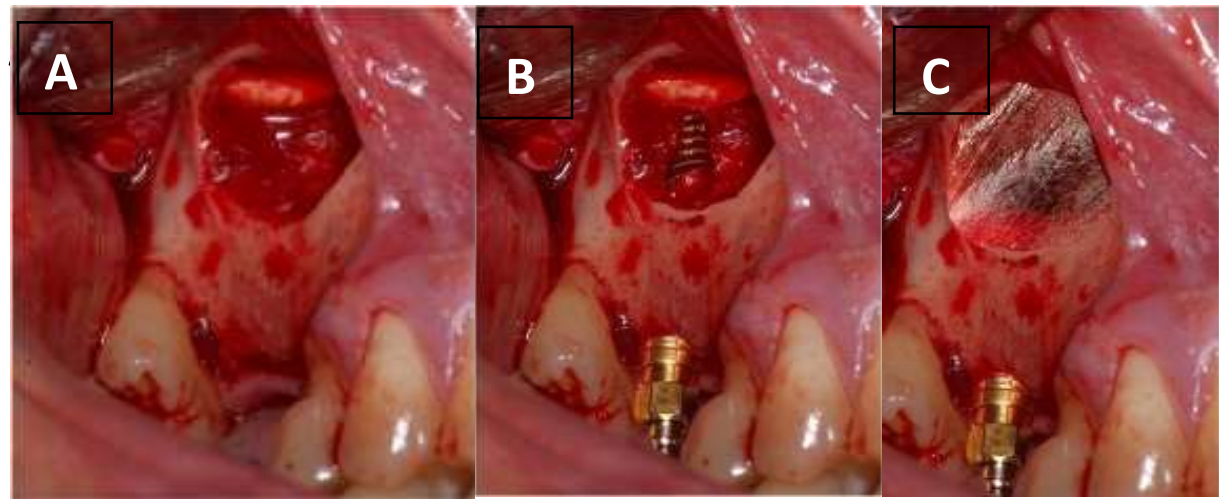

Fonte: Autores.

Após um período de 1 ano decorrido do procedimento cirúrgico as regiões foram reabertas (Figuras 4A e B) para instalação de componentes protéticos para finalizar a reabilitação do paciente. Foi realizado acompanhamento clínico e tomográfico desse paciente no período de 12 meses, sendo que as tomadas tomográficas foram feitas no pós-operatório imediato, 3, 6, 10 e 12 meses. No momento da reabertura os implantes encontravam-se estáveis e dessa forma as próteses do paciente foram instaladas. (Figura 4C).

Figura 4. A. Reabertura lado direito. B. Reabertura lado esquerdo. C. Aspecto oclusal da reabilitação protética.

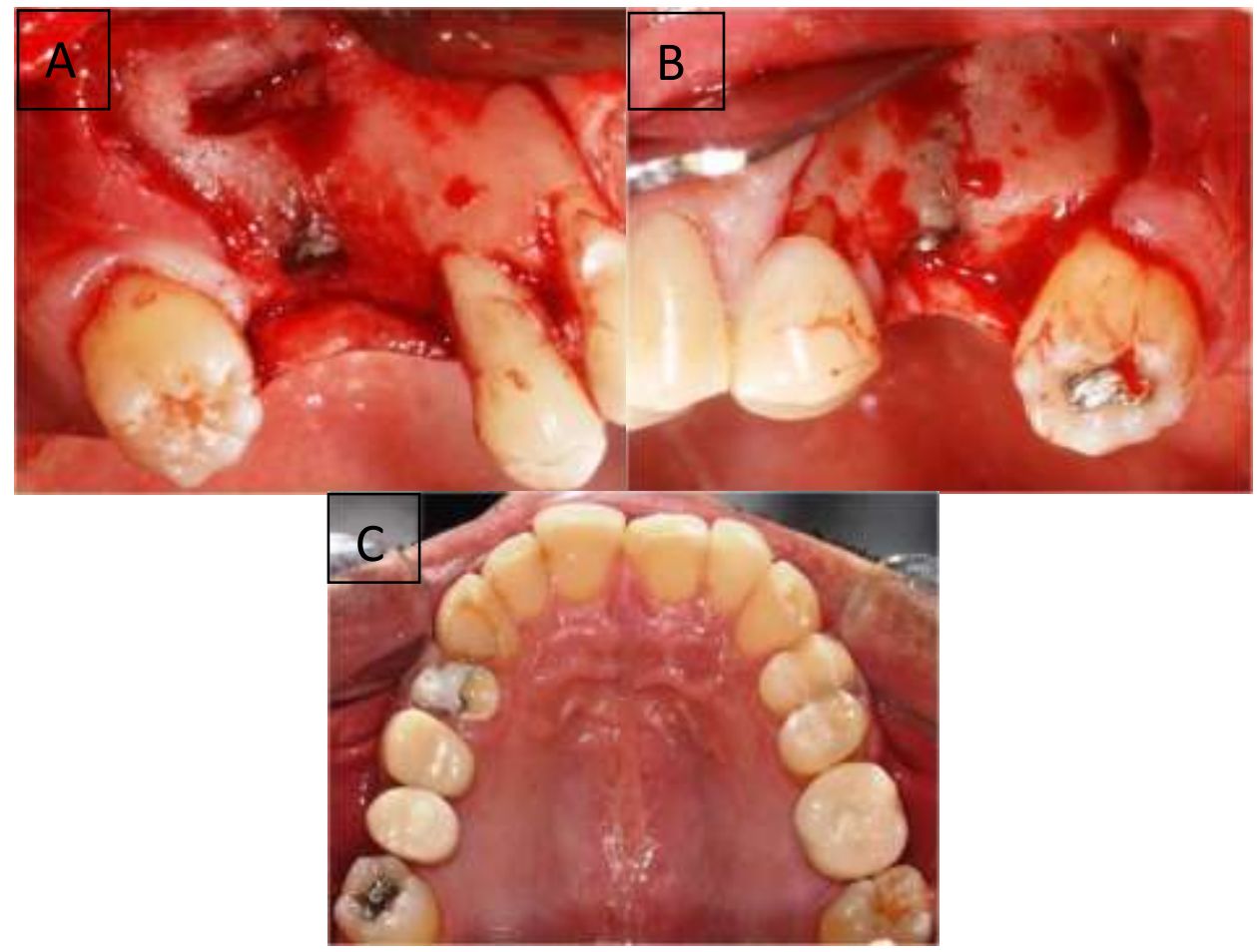

Fonte: Autores. 


\section{Análise Tomográfica}

Com o intuito de aferir a densidade óssea, tomografias computadorizadas com imagens obtidas por meio de um tomógrafo do tipo feixe cônico (Sirona Dental Systems GmbH, Bensheim, Germany), foram realizadas imediatamente após a instalação dos implantes e nos períodos de 3, 6, 10 e 12 meses pós-operatórios.

As Tomografias foram realizadas de forma padronizada. O paciente foi posicionado com o plano oclusal paralelo ao solo e o plano sagital mediano perpendicular ao solo, mantendo as configurações do cefalostato sempre constantes. Os fatores de aquisição para as tomografias foram constantes: $42 \mathrm{mAs}$, high contrast, $85 \mathrm{kV}$, espessura de corte de $0,3 \mathrm{~mm}$.

As imagens tomográficas foram analisadas por meio do software Galaxis versão 1.7 (Sirona Dental Systems GmbH, Bensheim, Germany) e os parâmetros de pós-processamento utilizados foram de espessura de corte de 0,3 mm, com espaçamento de $0,3 \mathrm{~mm}$.

\section{A seguir, sequência de imagens tomográficas:}

Figura 5. Tomografia Computadorizada Inicial.

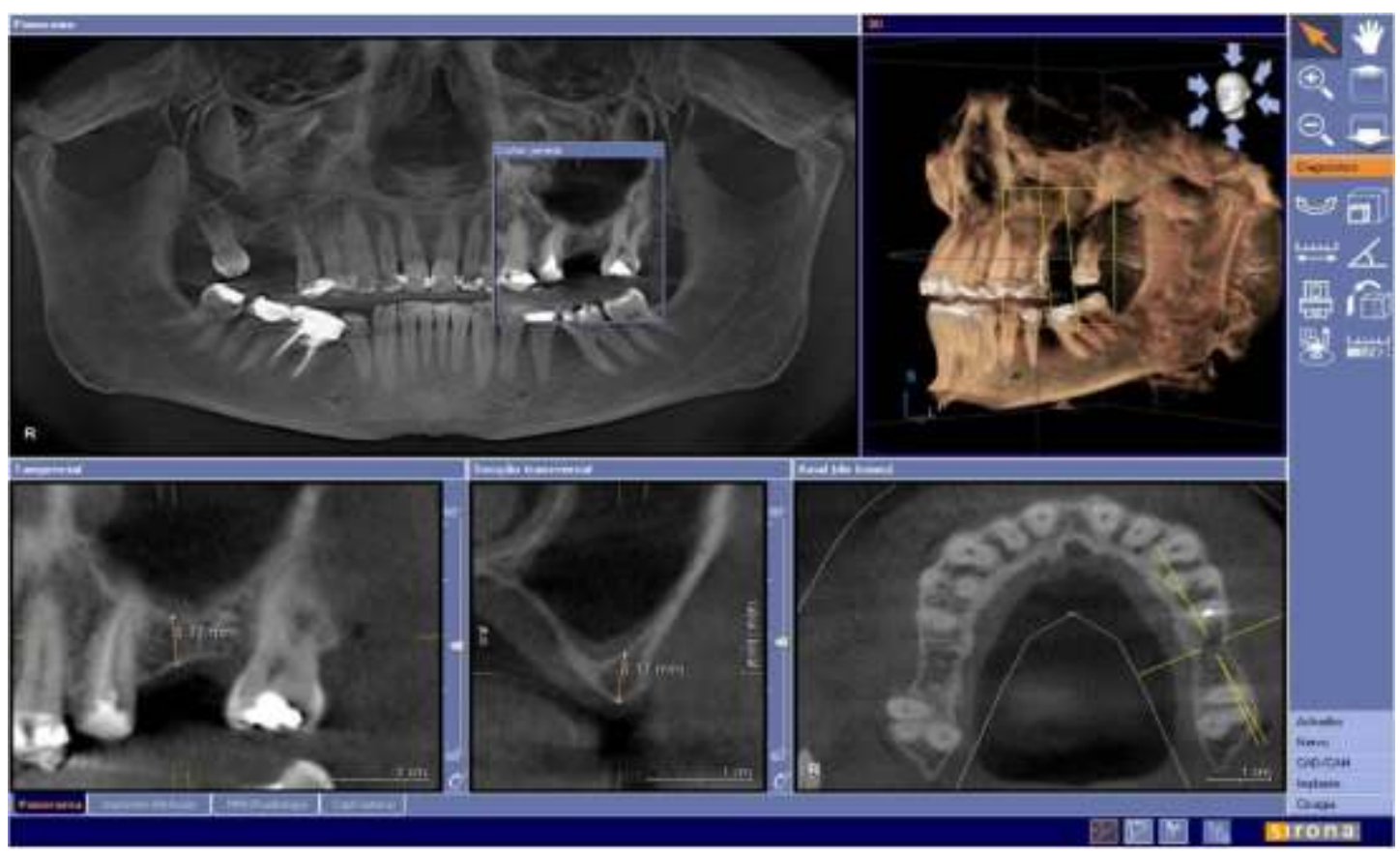

Fonte: Autores.

A Figura 6 apresenta a tomografia computadorizada pelas vistas frontal e sagital evidenciando a espessura óssea da região do elemento 26 (aproximadamente $5.17 \mathrm{~mm}$ ). 
Research, Society and Development, v. 10, n. 5, e33810514763, 2021

(CC BY 4.0) | ISSN 2525-3409 | DOI: http://dx.doi.org/10.33448/rsd-v10i5.14763

Figura 6. Tomografia Computadorizada.

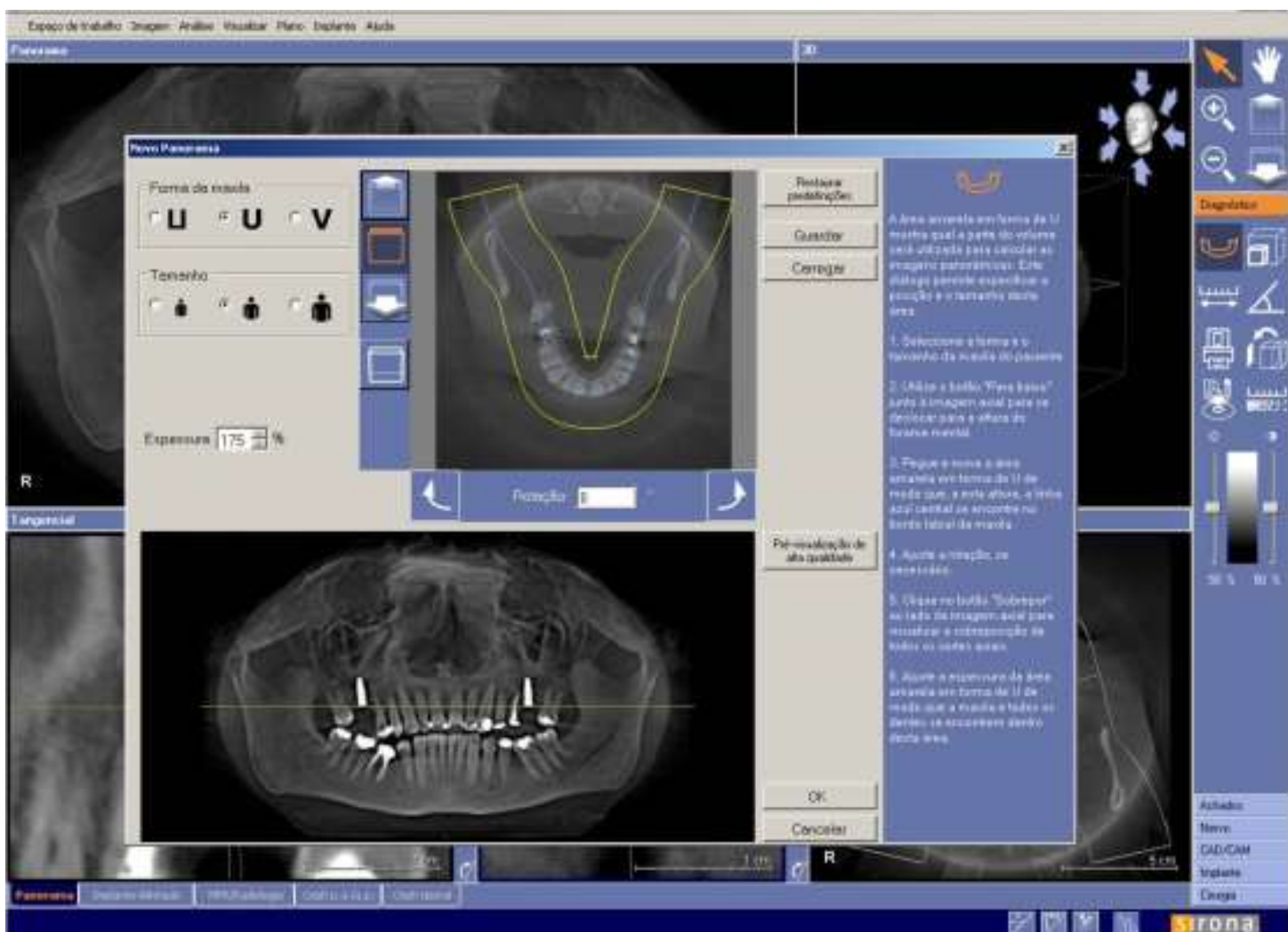

Fonte: Autores.

A Figura 7 mostra uma tomografia computadorizada do paciente já com ambos os implantes instalados.

Figura 7. Tomografia computadorizada.

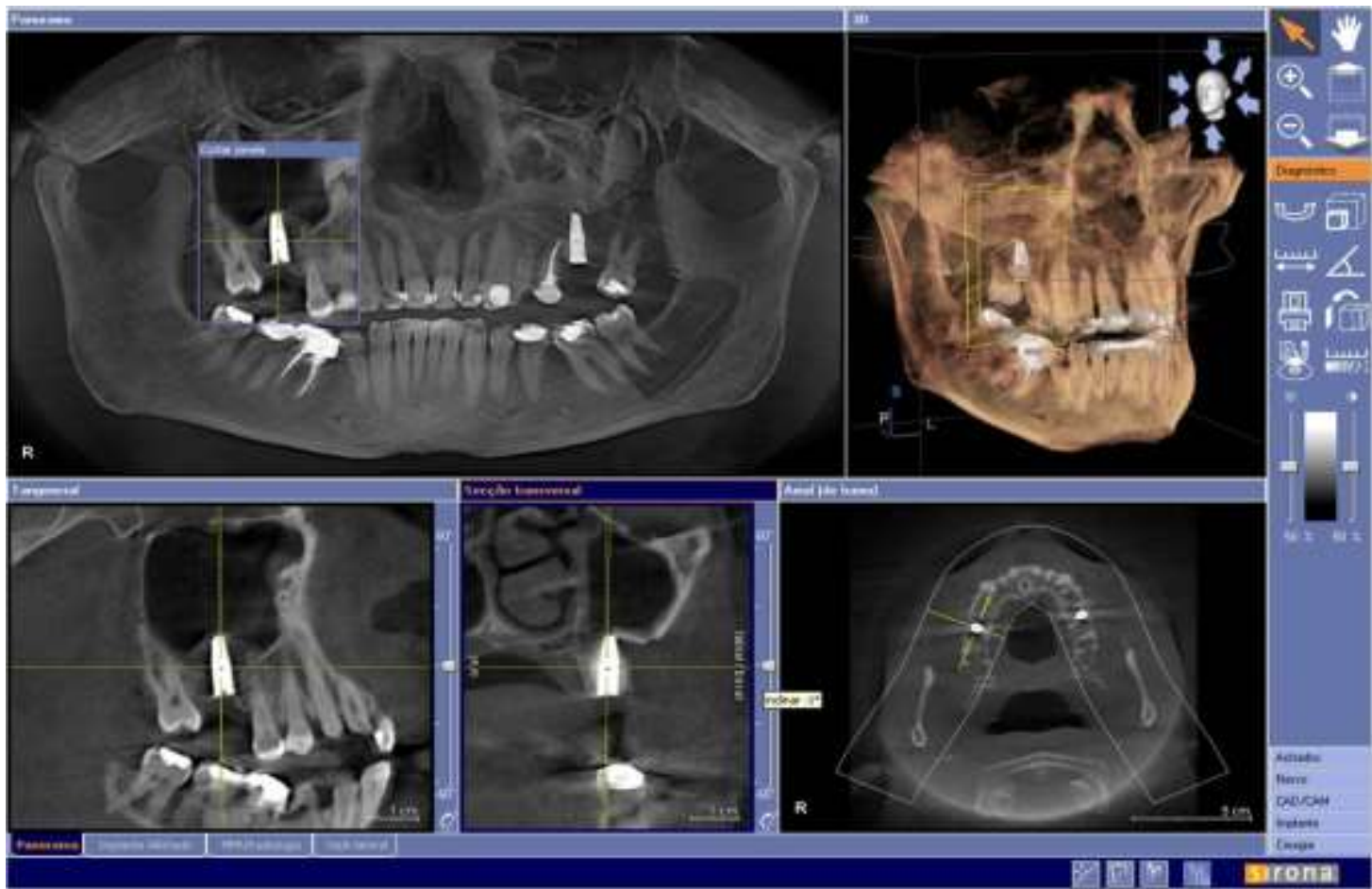

Fonte: Autores. 
Research, Society and Development, v. 10, n. 5, e33810514763, 2021

(CC BY 4.0) | ISSN 2525-3409 | DOI: http://dx.doi.org/10.33448/rsd-v10i5.14763

A Figura 8 apresenta uma tomografia computadorizada evidenciando a localização do implante na região do elemento 16 e suas dimensões.

Figura 8. Tomografia Computadorizada.

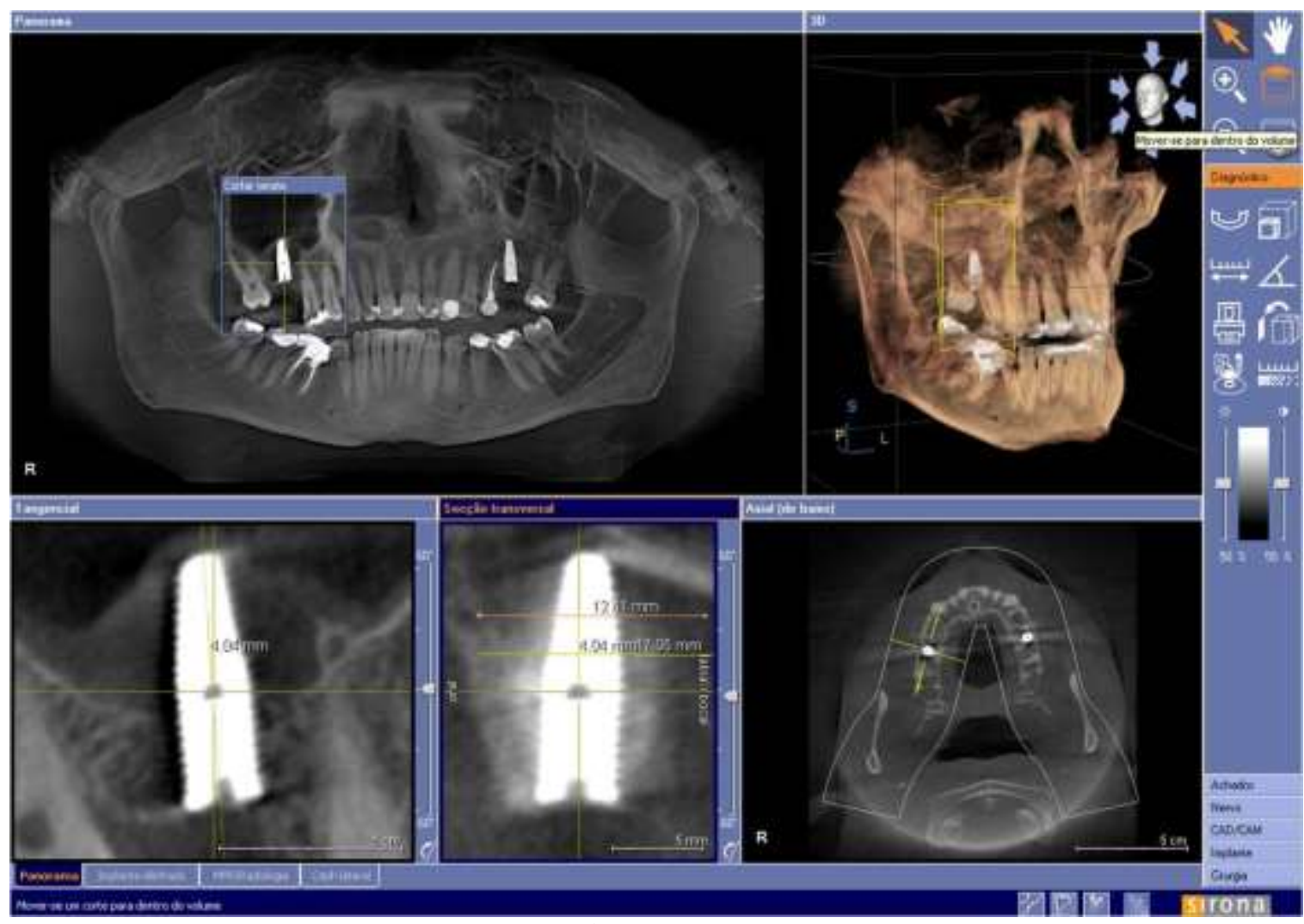

Fonte: Autores. 
Figura 9. Tomografia Computadorizada.

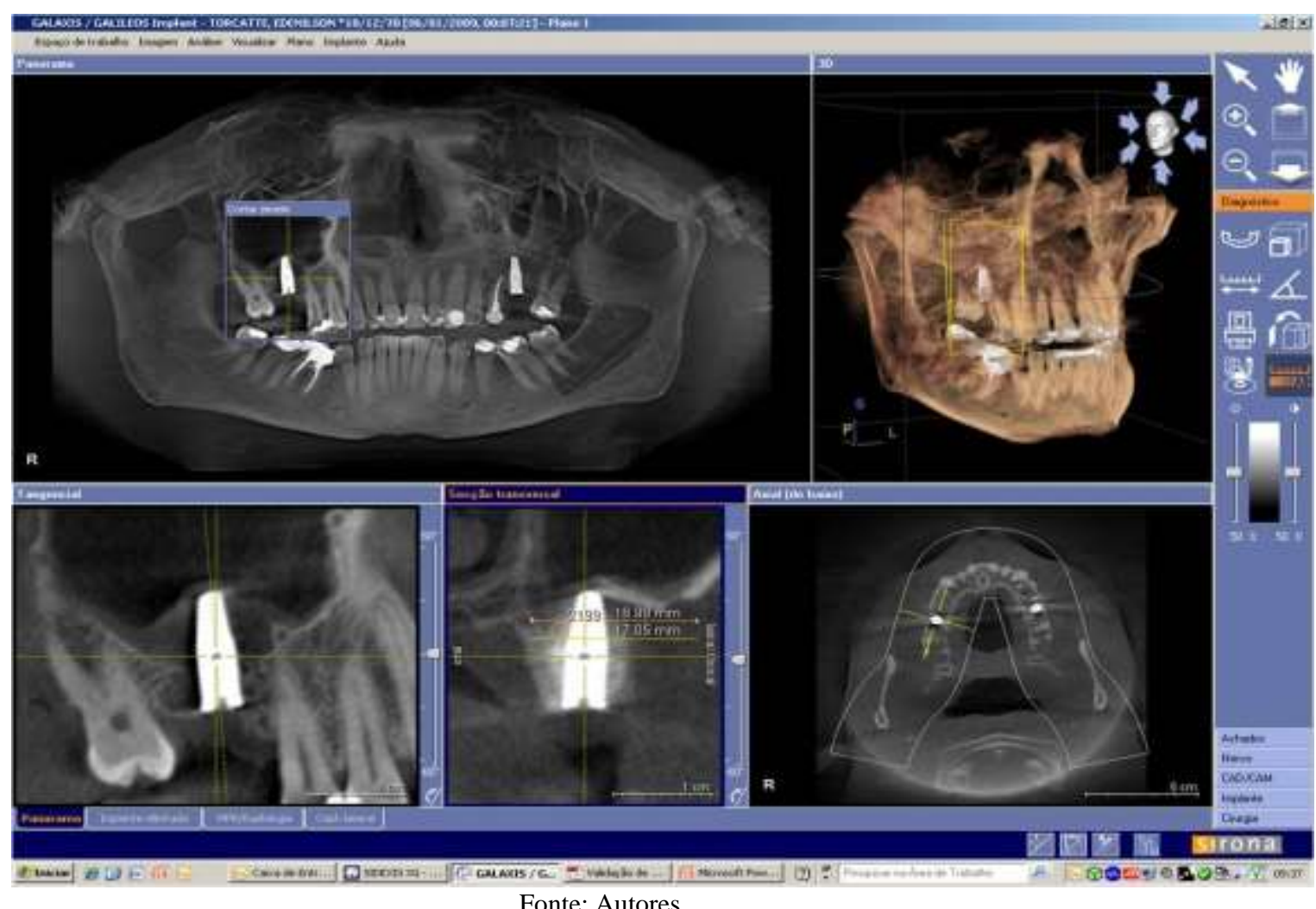

As Figuras 8 e 9 apresentam imagens tomográficas evidenciando a localização e as dimensões do implante instalado na região do elemento 16 .

\section{Medidas de densidade}

Para as mensurações de densidade ao redor dos implantes foram realizados os seguintes passos:

a) Abertura do programa Sidexis, em seguida do programa Galaxis;

b) Seleção e abertura da imagem da tomografia computadorizada;

c) Ajuste da curva panorâmica (para mandíbula) na janela "novo panorama".

O nível de referência para a reformatação da curva panorâmica foi padronizado na superfície da plataforma dos implantes. Os outros parâmetros como a "forma" e "tamanho" da mandíbula e porcentagem da espessura, também foram padronizados.

d) Ajuste da inclinação da imagem do implante.

Foi realizado o ajuste da inclinação da imagem do implante na janela "secção transversal" que corresponde ao corte parassagital.

e) Centralização da janela de visualização.

$\mathrm{Na}$ janela "secção transversal", por meio da ferramenta "reposicionar tracejado", posicionou-se a janela de visualização bem no centro da imagem do espaço interno do parafuso do implante.

f) Traçado da $1^{\mathrm{a}}$ linha de referência para medidas de densidade.

Por meio da ferramenta "medir comprimento", traçou-se uma reta horizontal $2 \mathrm{~mm}$ a partir do centro da janela de visualização.

g) Traçado da $2^{\mathrm{a}}$ linha de referência para medidas de densidade. 
Por meio da ferramenta "medir comprimento", traçou-se uma reta horizontal $4 \mathrm{~mm}$ a partir do centro da janela de visualização.

h) Obtenção das medidas de densidade.

As medidas de densidade foram realizadas ao redor do implante em áreas padronizadas, entre as linhas de referência. Para isso utilizou-se a ferramenta "visualizar valor de cinza".

D1P - medida de densidade 1 do lado palatino

D2P - medida de densidade 2 do lado palatino

D3P - medida de densidade 3 do lado palatino

D1V - medida de densidade 1 do lado vestibular

D2V - medida de densidade 2 do lado vestibular

D3V - medida de densidade 3 do lado vestibular

Todos os passos para a obtenção das medidas de densidade (D1P, D2P, D3P, D1V, D2V, D3V), ao redor dos implantes do lado direito (enxerto por coágulo) e esquerdo (enxerto autógeno) nas imagens tomográficas nos tempos inicial, 3 , 6, 10 e 12 meses após, foram realizados por um examinador calibrado.

Os valores das mensurações foram transferidos para o programa Microsoft ${ }^{\circ}$ Office Excel 2003 (Microsoft Corporation, USA) e submetidos à análise estatística.

\section{Análise de variação da Densidade em função dos Fatores Tempo e Tipo de Tratamento}

As análises estão divididas em duas partes: Longitudinais, onde é observada a evolução ao longo do tempo das medidas dos dois experimentos; e transversais, onde foram comparadas a cada etapa as medidas do lado direito com as do lado esquerdo (lado controle). Também são apresentados os resultados referentes a um teste de hipóteses.

A Tabela 1 apresenta os dados experimentais para os 12 pontos de observação. Foram tomadas 5 medidas de Densidade para cada ponto, ao longo de um ano.

Tabela 1. Dados Experimentais.

\begin{tabular}{|c|c|c|c|c|c|c|}
\hline \multicolumn{7}{|c|}{ PESQUISA ENXERTO ÓSSEO - MEDIDAS DE DENSIDADE } \\
\hline & & INICIAL & 3 MESES & 6 MESES & 10 MESES & 12 MESES \\
\hline \multirow{2}{*}{ D1P } & D1P-D & 2598 & 2570 & 2590 & 2191 & 2059 \\
\hline & D1P-E & 2052 & 2099 & 2149 & 1854 & 1841 \\
\hline \multirow{2}{*}{ D2P } & D2P-D & 2199 & 2144 & 2198 & 1960 & 1946 \\
\hline & D2P-E & 1921 & 1950 & 2018 & 1709 & 1735 \\
\hline \multirow{2}{*}{ D3P } & D3P-D & 1824 & 1843 & 1808 & 1449 & 1395 \\
\hline & D3P-E & 1967 & 1980 & 1942 & 1619 & 1446 \\
\hline \multirow{2}{*}{ D1V } & D1V-D & 2008 & 1875 & 2097 & 1836 & 1938 \\
\hline & D1V-E & 1836 & 1852 & 2130 & 1669 & 1566 \\
\hline \multirow{2}{*}{ D2V } & D2V-D & 1859 & 1895 & 1862 & 1523 & 1668 \\
\hline & D2V-E & 2003 & 2091 & 2337 & 2041 & 1841 \\
\hline \multirow{2}{*}{ D3V } & D3V-D & 1415 & 1749 & 1550 & 1349 & 1610 \\
\hline & D3V-3 & 1980 & 1997 & 2260 & 1830 & 1706 \\
\hline
\end{tabular}

Fonte: Autores. 
Na Tabela 2, são mostrados os valores médios, em cada etapa, para os dois experimentos realizados.

Tabela 2. Valores médios para cada conjunto de medidas.

\begin{tabular}{|c|c|c|c|c|c|c|}
\hline \multicolumn{7}{|c|}{ VALORES MÉDIOS DE DENSIDADE } \\
\hline & INICIAL & 3 MESES & 6 MESES & 10 MESES & 12 MESES & MÉDIA GERAL \\
\hline EXPERIMENTO & 1984 & 2013 & 2018 & 1718 & 1769 & 1900 \\
\hline CONTROLE & 1960 & 1995 & 2139 & 1787 & 1689 & 1914 \\
\hline
\end{tabular}

Fonte: Autores.

\section{Análises Longitudinais}

Nas análises longitudinais se observa a evolução da variável Densidade ao longo do tempo nos pontos em que foram aplicados os tratamentos sob comparação.

Para essa avaliação foram consideradas as médias das medidas de densidade D1P-D, D2P-D, D3P-D, D1V-D, D2VD e D3V-D, para cada instante de tempo, com relação ao lado de experimento. Para o lado de controle (Esquerdo), as médias foram calculadas de maneira análoga. O Gráfico 1 ilustra a evolução temporal da densidade média nos pontos de controle.

Gráfico 1. Médias das densidades para os grupos do Experimento e de Controle ao longo do tempo.

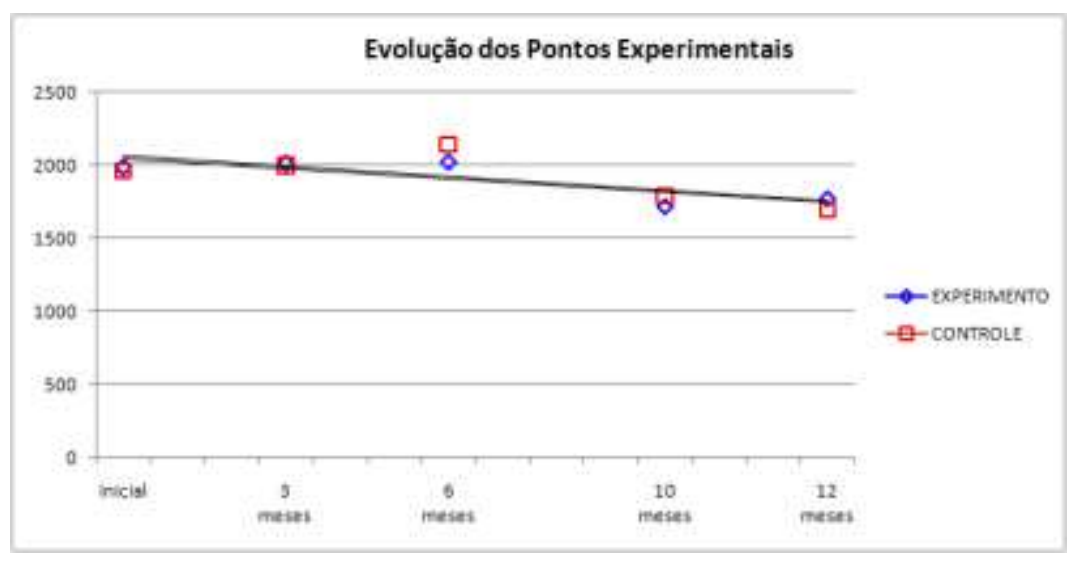

Fonte: Autores.

Observa-se que os dois conjuntos de medidas apresentam bastante semelhança, de tal forma que suas linhas de tendência se sobrepõem e indicam um decrescimento da densidade ao longo do tempo.

Quanto à evolução a cada observação, os dois conjuntos de medidas apresentam comportamentos semelhantes, com pequenas variações da primeira para a segunda e da segunda para a terceira medida. Do sexto para o décimo mês, acontece um decréscimo mais acentuado nos valores. Variações pequenas ocorrem nos 2 últimos meses de observação.

Ao longo de 1 ano, a densidade média decaiu por volta de $11 \%$ para o lado do experimento, e de $14 \%$ para o lado de controle. As variações são apresentadas em valores percentuais na Tabela 3.

Tabela 3. Variações de densidade ao longo do tempo.

\begin{tabular}{c|c|cccc|c}
\hline & Densidade & \multicolumn{4}{|c|}{ Variações Percentuais a cada medida } & \multicolumn{2}{c}{ Variação } \\
Lado & Inicial & $\mathbf{1}^{\mathbf{a}}$ para $\mathbf{2}^{\mathbf{a}}$ & $\mathbf{2}^{\mathbf{a}}$ para 3 & $\mathbf{3}^{\mathbf{a}}$ para 4 & $\mathbf{4}^{\mathbf{a}}$ para 5 & Geral \\
\hline EXPERIMENTO & 1984 & $+1,453 \%$ & $+0,240 \%$ & $-14,845 \%$ & $+2,988 \%$ & $-10,812 \%$ \\
CONTROLE & 1960 & $+1,786 \%$ & $+7,244 \%$ & $-16,469 \%$ & $-5,475 \%$ & $-13,811 \%$ \\
\hline
\end{tabular}




\section{Análises Transversais}

Nas análises transversais é feita a comparação entre as densidades médias do grupo controle e do grupo experimental, confrontando-se os resultados obtidos a cada etapa.

Também é realizado um teste estatístico, com o objetivo de verificar se o método de enxerto com sangue se diferencia do enxerto ósseo, no que diz respeito à densidade óssea média.

\section{Comparação a cada medida entre os diferentes tratamentos}

Ao comparar as médias a cada instante, verifica-se que as diferenças entre os valores são pequenas. As diferenças absolutas e percentuais são apresentadas na Tabela 4 , junto com os valores de média para cada conjunto de medidas.

Tabela 4. Diferenças entre os tratamentos.

\begin{tabular}{c|cccccc}
\hline & INICIAL & 3 MESES & 6 MESES & 10 MESES & 12 MESES MÉDIA GERAL \\
\hline EXPERIMENTO & 1984 & 2013 & 2018 & 1718 & 1769 & 1900 \\
CONTROLE & 1960 & 1995 & 2139 & 1787 & 1689 & 1914 \\
\hline Diferença & 24 & 18 & -122 & -69 & 80 & -14 \\
Diferença (\%) & $1,22 \%$ & $0,89 \%$ & $-5,69 \%$ & $-3,86 \%$ & $4,75 \%$ & $-0,72 \%$ \\
\hline
\end{tabular}

Fonte: Autores.

A maior diferença ocorreu seis meses após o procedimento, onde a densidade para o grupo Experimento foi 5,69 \% menor que a medida no grupo Controle. Estas informações dão uma indicação de que o tratamento Experimental pode ser tão efetivo quanto o tratamento Controle.

\section{Resultados do Teste de Hipóteses}

Foi realizado um Teste t para duas Amostras Pareadas, para verificar se o tratamento experimental é tão efetivo quanto o tratamento de referência. De acordo com os resultados do teste, ao considerar um nível de significância de 5\%, não há evidências de que as densidades médias sejam diferentes entre os dois tratamentos. Os dados do teste são apresentados na Tabela 5 . 
Tabela 5. Dados relativos à aplicação do teste de Hipóteses.

\begin{tabular}{lrr}
\hline & Controle & Experimento \\
\hline Média & 1914,03 & 1900,26 \\
Variância & 40561,34 & 112603,58 \\
Observações & 30 & 30 \\
Correlação de Pearson & 0,337 & \\
Hipótese da diferença de média & 0 \\
gl & 29 \\
Stat $\mathrm{t}$ & 0,2299 \\
$\mathrm{P}(\mathrm{T}<=\mathrm{t})$ uni-caudal & 0,4099 \\
$\mathrm{t}$ critico uni-caudal & 1,6991 \\
$\mathrm{P}(\mathrm{T}<=\mathrm{t})$ bi-caudal & 0,8198 \\
$\mathrm{t}$ critico bi-caudal & 2,0452 \\
\hline A diferença média entre as medidas de densidade $(\mathrm{M}=13,767 ; \mathrm{DP}=327,97 ; \mathrm{N}=30$ ) \\
foi pequena; $\alpha=0,05 ; \mathrm{t}(29)=0,2299 ; \mathrm{P}(\mathrm{T}<=\mathrm{t})$ bi-caudal $=0,820 ;$ este valor-P não \\
formece evidências de que as médias sejam diferentes entre os dois tratamentos.
\end{tabular}

Fonte: Autores.

Nas análises Longitudinais, verificou-se que há um decaimento da ordem de 10 a 15\% na densidade óssea após o período de um ano.

Com relação às análises Transversais, observa-se que os dois tratamentos apresentam medidas semelhantes de densidade óssea ao longo do tempo, dando indicações de que o tratamento experimental pode ser tão efetivo quanto o tratamento de controle.

\section{Discussão}

Neste caso clínico, constatando-se a necessidade existente e levando em consideração a presença de condições favoráveis à realização de levantamento de seio bilateral com instalação imediata de implantes, decidiu-se efetuar o preenchimento de um dos seios apenas com coágulo e o outro com osso autógeno particulado, visando a realização de uma análise comparativa entre os resultados das técnicas. No período pós-operatório imediato, o paciente não relatou dor nas regiões em que recebeu os implantes dentários e onde foi efetuado levantamento de seio maxilar. As tomografias realizadas durante 1 ano revelaram neoformação óssea nos dois seios maxilares, com e sem enxerto ósseo, em volta dos implantes e a definição de novos limites nos seios maxilares. Foi possível visualizar claramente que, durante todo o período de acompanhamento, a janela óssea elevada juntamente com a membrana sinusal se tornou o novo assoalho.

Kim (2010) e colaboradores, afirmam que os coágulos sanguíneos não têm integridade suficiente para permitir que a membrana sinusal permaneça em uma posição elevada por períodos de tempo terapeuticamente eficazes, visto que em seu estudo experimental realizado em cadelas adultas que tiveram o espaço entre a membrana e o assoalho do seio preenchido por sangue venoso retirado de cada cão, o espaço criado colapsou e a membrana do seio caiu sobre o implante. Em contrapartida, em nosso estudo não foi observado colabamento da membrana sobre o implante, confirmando que o coágulo pode ser considerado um material de preenchimento satisfatório.

A utilização do coágulo sanguíneo como material de preenchimento para levantamento da membrana sinusal elimina a necessidade de uma área doadora (Chen et al., 2007; Kim et al., 2010; Falah et al., 2016). A redução do tempo cirúrgico, da morbidade, e do custo dos procedimentos, são vantagens que direcionam a escolha para essa nova técnica, desde que 
respeitadas suas limitações (Thor et al., 2007; Betoni Junior et al., 2012; Falah et al., 2016; Fouad et al., 2018; Bassi et al., 2015; Ferreira et al., 2021).

Muitos substitutos ósseos estão disponíveis para preencher as áreas em que a membrana sinusal é elevada, porém poucos tornam possível a formação de osso vital e bem estruturado. A escolha do osso autógeno como material de preenchimento do seio esquerdo foi feita pelo fato de ser a mesma considerada "padrão ouro" em reconstruções ósseas para reabilitações com implantes dentários. Características como osteoindução, osteocondução, osteogênese e osteopromoção, asseguram a previsibilidade positiva de reabsorção do material enxertado e a substituição por tecido ósseo vital. Apesar de sua biocompatibilidade, o fato do osso autógeno requerer uma área doadora se torna negativo quanto à morbidade, o tempo cirúrgico e os possíveis acidentes trans operatórios (Ferreira et al., 2021; Guilherme et al., 2009; Kürrkçü et al., 2005; Lundgren et al., 2004; Srouji et al., 2009).

Lundgren et al., (2003), após realizar cistotomia no seio maxilar, confirmou a formação espontânea de osso entre a parede lateral e a membrana do seio, utilizando coágulo sanguíneo como único material de preenchimento. A pesquisa envolveu 10 pacientes, nos quais foram instalados 19 implantes. Os autores relatam que o compartimento segregado criado pela elevação da membrana do seio, a estabilidade dos implantes e da janela óssea de substituição, permitiram a formação óssea de acordo com o princípio de regeneração óssea guiada. Em 2004, o mesmo autor apresentou uma nova técnica para a formação óssea no seio maxilar utilizando o coágulo como único material de preenchimento (Lundgren et al., 2004).

Nossos resultados corroboram com os achados de Falah (2016) e colaboradores em um experimento no qual 30 procedimentos de levantamento de seio nasal sem enxerto e 72 implantes dentários colocados em 18 pacientes consecutivos, utilizando a abordagem de janela lateral foram executados. Com base nos dados histológicos e hitomorfométricos em relação ao tecido ósseo vital neorformado dos 72 implantes instalados, concluíram que o coágulo sanguíneo pode ser considerado material de enxerto osteogênico autólogo, para o qual os osteoprogenitores podem migrar, se diferenciar e regenerar o osso.

\section{Considerações Finais}

A realização do levantamento do seio maxilar sem o uso de osso autógeno particulado mostrou-se uma possibilidade viável para a indução de neoformação óssea e sucesso do tratamento na instalação imediata de implantes. A utilização apenas do coágulo sanguíneo como material de preenchimento permitiu neoformação óssea com densidade satisfatória, além de redução da morbidade e dos custos do procedimento quando comparada com a técnica convencional que utiliza osso autógeno e necessita de uma área doadora. Novos estudos devem ser realizados para que possa ser analisada com cuidado a estabilidade à longo prazo do osso aumentado.

\section{Referências}

Albuquerque, A. F., Cardoso, I., da Silva, J., Germano, A., Dantas, wagner, \& Gondim, A. (2014). Levantamento de Seio maxilar utilizando osso liofilizado associado a instalação imediata de implante do tipo cone morse: relato de caso. Revista Da Faculdade De Odontologia - UPF, 19(1).

Bassi, A. P., Pioto, R., Faverani, L. P., Canestraro, D., \& Fontão, F. G. (2015). Dental implant placement after maxillary sinus lift without a graft: a feasible option. International journal of oral and maxillofacial surgery, 44(12), 1583-1584.

Bassi, A. P., Pioto, R., Faverani, L. P., Canestraro, D., \& Fontão, F. G. (2015). Maxillary sinus lift without grafting, and simultaneous implant placement: a prospective clinical study with a 51-month follow-up. International journal of oral and maxillofacial surgery, 44(7), 902-907.

Betoni Junior, W., Esteves, J. C., \& Queiroz, T. P. (2012). Levantamento de seio maxilar com enxerto de coágulo sanguíneo / Sinus lifting associated to blood clot grafting. A report of two clinical cases. Implant News, 9(2), 249-254.

Burchardt, H. (1983). The biology of bone graft repair. Clin Orthop Relat Res., (174), 28-42.

Chen, T. W., Chang, H. S., Leung, K. W., Lai, Y. L., \& Kao, S. Y. (2007). Implant placement immediately after the lateral approach of the trap door window procedure to create a maxillary sinus lift without bone grafting: A 2- Year retrospective evaluation of 47 implants in 33 patients. Oral Maxillofac Surg., $65(11), 2324-2328$. 
David, G. M., Vermudt, A., Ghizoni, J. S., Pereira, J. R., \& Pamato, S. (2018). Levantamento de seio maxilar: Uma comparação de técnicas. Journal of Research in Dentistry, 6(2), 43-48.

Falah, M., Sohn, D. S., \& Srouji, S. (2016). Graftless sinus augmentation with simultaneous dental implant placement: clinical results and biological perspectives. International journal of oral and maxillofacial surgery, 45(9), 1147-1153.

Ferreira, P. H. S. G., Bizelli, V. F., Bittencourt Junior, D., Fontão, F. G. K., \& Bassi, A. P. F. (2021). Avaliação tomográfica e histológica da técnica de levantamento de seio maxilar e instalação de implantes dentários sem utilização de biomaterial com acompanhamento de 36 meses: Relato de caso. Research, Society and Development, 10(2), e31810212558.

Fouad W., Osman A., Atef M., \& Hakam M. (2018). Elevação guiada do assoalho do seio maxilar usando osso bovino desproteinizado versus elevação da membrana Schneideriana sem enxerto com colocação simultânea de implante: ensaio clínico randomizado. Clin Implant Dent Relat Res., 20(3),424-433.

Guilherme, A. S.; Zavanelli, R. A.; Fernandes, J. M. A.; Castro, A. T.; Barros, C. A.; \& Souza, J. E. A. (2009). Implantes osseointegráveis em áreas com levantamento do seio maxilar e enxertos ósseos. RGO., 57(2), 157-163.

Kim H. R., Choi B. H., Xuan F., \& Jeong S. M. (2010). The use of autologous venous blood for maxillary sinus floor augmentation in conjunction with sinus membrane elevation: an experimental study. Clin. Oral Impl. Res., 21(3), 346-349.

Kürrkçü, M., Oz, I. A., Köksal, F., Benlidayi, E., \& Günesli, A. (2005). Microbial Analysis of the Autogenous Bone Collected by Bone Filter During Oral Surgery: A Clinical Study. Journal of Oral and Maxillofacial Surgery., 63(11), 1593-1598.

Lundgren, S., Andersson, S., Gualini, F., \& Sennerby, L. (2004). Bone reformation with sinus membrane elevation: a new surgical technique for maxillary sinus floor augmentation. Clin Implant Dent Relat Res., 6(3), 165-173.

Lundgren, S., Andersson, S., \& Sennerby, L. (2003). Spontaneous bone formation in maxillary sinus after removal of a cyst: Coincidence or consequence? Clin Implant Dent Relat Res., 5(2), 78-81.

Patel, S., Lee, D., Shiffler, K., Aghaloo, T., Moy, P., \& Pi-Anfrus, J. (2015). Resonance Frequency Analysis of Sinus Augmentation by Osteotome Sinus Floor Elevation and Lateral Window. Journal of Oral and Maxillofacial Surgery, 73(10), 1920-1925.

Pereira, A. S., Shitsuka, D. M., Parreira, F. J., \& Shitsuka, R. (2018). Metodologia da Pesquisa Científica. UFSM.

Srouji, S., Kizhner, T., Ben David, D., Riminucci, M., Bianco, P., \& Livne, E. (2009). The Schneiderian membrane contains osteoprogenitor cells: in vivo and in vitro study. Calcified tissue international, 84(2), 138-145. https://doi.org/10.1007/s00223-008-9202-x

Terra, R. C., Betoni Júnior, W., Luvizuto, E. R., \& Queiroz, T. P. (2011). Aspectos biológicos da regeneração óssea em levantamento de seio maxilar / Biological aspects of bone regeneration in maxillary sinus lifting. ImplantNews., 8(6), 807-811.

Thor, A., Sennerby, L., Hirsch, J. M., \& Rasmusson, L. (2007). Bone formation at the maxillary sinus floor following simultaneous elevation of the mucosal lining and implant installation without graft material: an evaluation of 20 patients treated with 44 Astra Tech implants. Journal of oral and maxillofacial surgery: official journal of the American Association of Oral and Maxillofacial Surgeons, 65(1), 64-72. 